\title{
ÉTUDE DE LA BIOSYNTHESE ET DE L'UTILISATION DES ACIDES GRAS GHEZ LA POULE PONDEUSE
}

\author{
B. LECLERCQ \\ avec la collaboration technique de Marie-Rose SALichon et de P. Doucet \\ Station de Recherches avicoles, \\ Centre de Recherches de Tours, I. N. R. A., \\ 37 - Nouzilly \\ RÉSUMÝ
}

Soixante-dix pour cent du carbone de l'acide palmitique de l'œuf provient directement soit des glucides, soit de l'acide palmitique alimentaires. L'utilisation de ce dernier pour la synthèse de lipides vitellins ou adipeux est particulièrement élevée. Celle des glucides pour la lipogenèse est très réduite si on incorpore à l'aliment $\mathrm{I} 5 \mathrm{p}$. roo de matière grasse. La Poule dépose chaque jour dans ses réserves corporelles presque autant d'acides gras que dans son cuf. La relation entre l'intensité de ces deux dépôts n'est pas linéaire; en cas de lipogenèse intense, la part destinée aux réserves adipeuses s'accroît plus vite que la part destinée à l'œuf.

La quantité de lipides contenue dans la carcasse est directement proportionnelle à l'intensité des dépôts journaliers dans les réserves corporelles. Les phénomènes de lipolyse doivent donc être réduits à ce niveau d'autant que les acides gras des réserves ne participent que pour une faible part à la vitellogenèse.

La Poule utilise préférentiellement les acides gras de l'aliment pour la synthèse du vitellus. Les conversions entre acides gras sont faibles. Elles ont essentiellement lieu dans le sens d'un allongement de la chaîne carbonée et de la désaturation.

\section{INTRODUCTION}

Les acides gras, en particulier ceux que l'organisme animal est incapable de synthétiser, peuvent être incorporés plus ou moins largement dans les lipides de l'œuf en fonction de l'apport alimentaire. Il en est ainsi par exemple de l'acide linoléique (FEIGENbaum et Fisher, I959; WhEELER et al., I959; MurTy et Reiser, I964), de l'acide linolénique (MURTY et REISER, I96I) et de l'acide élaïdique (LeCleERCQ, I965, KaUfman et Mankel, I967). Ceci laisse supposer que les acides gras de 1'œuf proviennent directement de l'aliment et que la contribution des réserves grasses est faible (ALMQUIST, I934; LECLERCQ, I967). Certains travaux cependant 
conduisent à attribuer à ces réserves corporelles un rôle non négligeable Ainsi, pour HEALD et al., (rg63) l'élévation de la concentration sanguine en acides gras libres lors de l'entrée en ponte traduit l'effort de mobilisation des réserves en vue de la vitellogenèse. Des conclusions voisines sont tirées à la suite d'expériences de carence en acide linoléique de la poule pondeuse (REISER, I950; BALNAVE et Brown, I968).

Dans le présent travail, nous cherchons à préciser l'origine des acides gras de l'œuf en nous intéressant spécialement à ceux dont la poule peut réaliser la synthèse à partir soit des glucides, soit des matières grasses alimentaires.

\section{MATÉRIEL E'T MÉTHODES}

\section{Essai no 1}

Dans un premier essai, on détermine l'origine de l'acide palmitique vitellin en précisant les parts respectives qui reviennent à l'acide palmitique du régime (régime gras) ou aux glucides alimentaires (régime gras et régime lipidoprive). On utilise 16 poules âgées de 34 semaines, logées

TABLEAU I

Composition des régimes

(en p. Ioo)

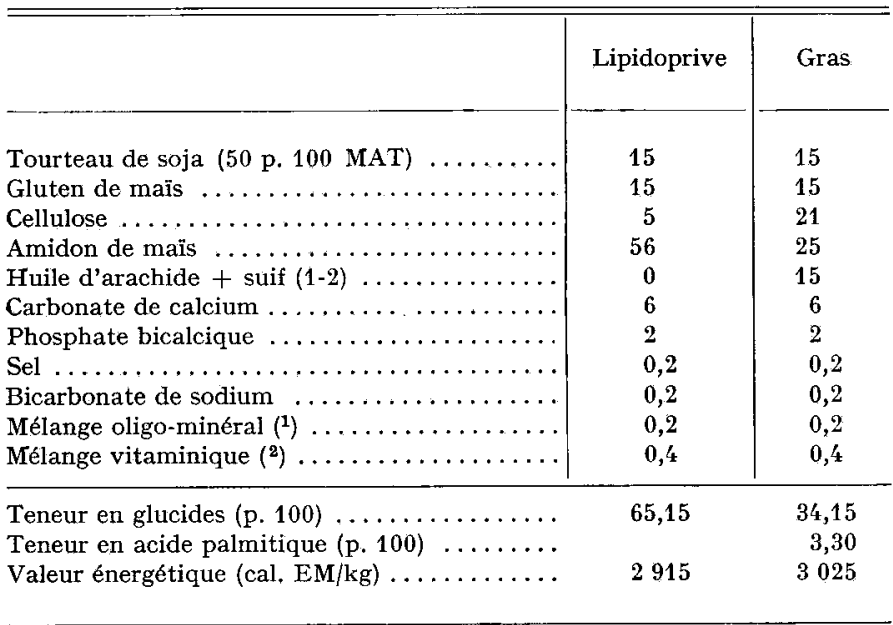

(1) Composition du mélange oligo-minéral (pour $100 \mathrm{~kg}$ régime) : protoxyde anhydre de manganese : $14 \mathrm{~g}$; oxyde de zinc: $10 \mathrm{~g}$; sulfate ferreux : $14 \mathrm{~g}$; sulfate de cuivre : $1,1 \mathrm{~g}$; carbonate de cobalt : $0,06 \mathrm{~g}$; iodure de potassium : $0,2 \mathrm{~g}$; carbonate de calcium : $150 \mathrm{~g}$.

$\left({ }^{2}\right)$ Composition du mélange vitaminique (pour $100 \mathrm{~kg}$ régime) : vitamine $\mathrm{A}: 800000 \mathrm{UI}$; vitamine $\mathrm{D}_{3}: 100-000 \mathrm{UI}$; riboflavine $: 2 \mathrm{~g} ; \alpha$-tocophérol : $1 \mathrm{~g}$; BHT : $10 \mathrm{~g}$; DL-méthionine : $50 \mathrm{~g}$; cyanocobalamine : $3 \mathrm{mcg}$; glucose : $314 \mathrm{~g}$. 
en cages individuelles, présentant un poids vif et exportant en moyenne chaque jour un poids d'œuf aussi semblables que possible. Dix d'entre elles reçoivent le régime gras et six le régime lipidoprive pendant une période d'adaptation de $\mathrm{r}$ mois. La composition des régimes fait l'objet du tableau I. La composition en acides gras de la matière grasse est rapportée dans le tableau 2.

\section{TABLEAU 2}

Composition en acides gras du mélange huile d'arachide et de suif

(p. roo des acides gras)

\begin{tabular}{c|r}
\hline Acides gras & P. 100 \\
\hline $14: 0$ & \\
$16: 0$ & 3,2 \\
$16: 1$ & 24,1 \\
$18: 0$ & 1,8 \\
$18: 1$ & 18,2 \\
$18: 2$ & 44,5 \\
& 6,2 \\
\hline
\end{tabular}

L'expérience proprement dite dure 48 heures. Soixante-huit $\mu \mathrm{Ci}$ de glucose U-C ${ }^{14}$ (CEA) ou $47 \mu \mathrm{Ci}$ d'acide palmitique-I-C14 (CEA) sont distribués à chaque animal au cours de ces deux jours sous forme de 8 gélules, à raison de 4 par jour. Les distributions ont lieu à 7 , Ir, I5 et Ig heures. Le premier jour; les animaux reçoivent leur aliment à 7 heures du matin après un jeâne de ro heures. Simultanément, on leur fait ingérer une gélule de noir Crésol, une gélule d'antibiotiques (30 mg de dihydrostreptomycine, I2 o0o UI de benzylpénicillinate de sodium et I6 ooo UI de benzylpénicillinate de procaïne) et la première gélule de précurseur radioactif. Le colorant lipophile permet de repérer le vitellus synthétisé au début de l'expérience. Le mélange antibiotique a pour rôle de limiter les modifications du glucose libre (précurseur radioactif) par la flore du tube digestif pour que, de ce point de vue, la destinée du marqueur soit aussi proche que possible de celle des polysaccharides digestibles. A I9 heures, le premier jour, on peut estimer que les divers pools métaboliques du foie et du sang ont atteint leur état de saturation maximum en carbone radioactif, étant donné le turnover rapide des constituants lipidiques et glucidiques de ces compartiments. Il doit en être de même du vitellus formé à cet instant, puisqu'il tire son origine du foie. On fait donc ingérer aux poules une gélule de colorant rouge écarlate pour repérer ce vitellus. Le lendemain, les animaux reçoivent les gélules de précurseur radioactif aux heures habituelles, une gélule d'antibiotiques à 7 heures et une gélule de colorant rouge à I9 heures. Ils sont mis à jeun vers $2 \mathrm{I}$ heures et sacrifiés le lendemain matin à 7 heures par section des veines jugulaires. La grappe ovarienne est aussitôt prélevée et coagulée à l'eau bouillante pendant dix minutes. On ne recueille par grattage que le vitellus coloré en rouge. Le reste ainsi que les œufs pondus durant deux jours sont rassemblés en vue d'analyse. Le tube digestif est également prélevé ainsi que les fèces afin de déterminer les constituants radioactifs non digérés. La carcasse est broyée, lyophilisée et conservée pour analyse. Enfin, on enregistre les consommations individuelles d'aliment en prenant soin d'éviter tout gaspillage.

\section{Essai $n^{\circ} 2$}

Vingt et une poules sont réparties en 7 groupes d'égale importance. Trois de ces groupes reçoivent le régime lipidoprive et quatre autres le régime gras. Les poules appartenant à un groupe ingérant le régime lipidoprive et à un groupe ingérant le régime gras reçoivent $50 \mu \mathrm{Ci}$ d'acide palmitique-I-14C ; il en est de même avec 1 'acide oléique-ro-14C et le glucose-U-14C. Seuls les animaux d'un groupe consommant le régime gras ingèrent de l'acide stéarique-I-14C. La distribution des molécules marquées se fait sous forme de 8 gélules distribuées en une seule journée à $6,8,10,12,14,16,18$ et 20 heures. A I2 et 20 heures, on leur fait absorber également une gélule de colorant noir Crésol et à 6 heures, une gélule d'antibiotiques. Le sacrifice a lieu le len- 
demain matin à 6 heures. Après cuisson de la grappe ovarienne, on recueille le vitellus coloré en noir. Au cours des deux essais, les acides gras radioactifs sont distribués en solution dans de l'huile de tournesol à raison de $100 \mathrm{mg}$ par gélule.

\section{Méthodes d'analyse}

Les lipides sont extraits par la méthode de Folch. Préparés par le mélange méthanol-acide sulfurique (95-5), les esters méthyliques sont purifiés sur couche mince préparative de silica gel G et analysés en chromatographie gaz-liquide sur DEGS. La radioactivité est mesurée par scintillation liquide dans le toluène additionné de $4 \mathrm{~g}$ de PPO et $50 \mathrm{mg}$ de POPOP par litre de solvant. L'activité spécifique de chaque acide gras est déterminée de la façon suivante. On mesure d'abord l'activité spécifique du mélange des esters méthyliques. Puis on analyse la répartition de la radioactivité entre les acides saturés, les acides mono-insaturés et les acides polyinsaturés (acide arachidonique) après séparation par chromatographie en couche mince sur silicagel imprégné de nitrate d'argent. Enfin, la répartition de la radioactivité au sein de chaque classe d'acides gras est déterminée après séparation de chacun d'eux par chromatographie gaz-liquide préparative ; les contaminations entre pics sont ainsi extrêmement réduites puisqu'en injectant séparément les esters d'acides saturés et les esters d'acides mono-insaturés, on obtient des pics très bien séparés les uns des autres. Connaissant la composition en acides gras du mélange analysé, on peut déduire facilement l'activité spécifique de chaque acide gras.

\section{TABLEAU 3}

Incorporation des précurseurs marqués dans le vitellus et les graisses de réserves (essai $n^{0} \mathrm{I}$ )

\begin{tabular}{|c|c|c|c|}
\hline \multirow[b]{2}{*}{ Marqueur utilisé } & \multirow{2}{*}{$\frac{\text { Régime lipidoprive }}{\text { Glucose-U. }{ }^{14} \mathrm{C}}$} & \multicolumn{2}{|c|}{ Régime gras } \\
\hline & & Glucose $\mathrm{U}^{14} \mathrm{C}$ & Ac. palmit. ${ }^{-1-14} \mathrm{C}$ \\
\hline a) Quantité de précurseur ingérée ( ${ }^{1}$ ) (g) & $135,9 \pm 12,7$ & $71,4 \pm 1,8$ & $6,81 \pm 0,15$ \\
\hline b) Énergie métabolisable ingérée (Cal/48 h) .. & 610 & 634 & 623 \\
\hline c) d.p.m. ingérées (10 d.p.m.) $\ldots \ldots \ldots \ldots$ & $150,7 \pm 6,7$ & $152,1 \pm 7,3$ & $104,6 \pm 2,55$ \\
\hline $\begin{array}{l}\text { d) Activité spécifique du carbone du précur- } \\
\text { seur (d.p.m./mg) } \ldots \ldots \ldots \ldots \ldots \ldots \ldots \ldots\end{array}$ & $2895 \pm 313$ & $5278 \pm 224$ & $22153 \pm 3870$ \\
\hline $\begin{array}{l}\text { e) Activité spécifique du carbone de l'acide } \\
\text { palmitique vitellin (d.p.m./mg) } \ldots \ldots \ldots\end{array}$ & $1945 \pm 174$ & $1009 \pm 168$ & $9445 \pm 668$ \\
\hline f) Valeur du rapport $\frac{e}{d} \times 100 \ldots \ldots \ldots \ldots$ & $68,9 \pm 5,7$ & $18,7 \pm 2,6$ & $45,3 \pm 4,7$ \\
\hline g) $\frac{\text { d.p.m. des acides gras vitellins }}{\text { d.p.m. ingérées }} \times 100 \ldots$ & $4,40 \pm 0,35$ & $1,20 \pm 0,19$ & $14,6 \pm 1,9$ \\
\hline $\begin{array}{l}\text { h) Quantité d'acides gras vitellins synthétisés } \\
\text { en } 48 \text { h à partir du précurseur (g) } \ldots \ldots \text {. }\end{array}$ & $3,40 \pm 0,47$ & $0,48 \pm 0,07$ & $0,97 \pm 0,09$ \\
\hline i) $\frac{\text { d.p.m. des acides gras de réserves }}{\text { d.p.m. ingérées }} \times 100 \ldots$ & $1,77 \pm 0,51$ & $1,38 \pm 0,28$ & $12,3 \pm 5,6$ \\
\hline $\begin{array}{l}\text { j) Quantité d'acides gras de réserves synthé- } \\
\text { tisés en } 48 \text { h à partir du précurseur (g) }\end{array}$ & $1,46 \pm 0,51$ & $0,55 \pm 0,10$ & $0,89 \pm 0,39$ \\
\hline
\end{tabular}

(1) Pour le glucose, il s'agit du glucose-14 $\mathrm{C}$ et du glucose des glucides alimentaires. Pour l'acide palmitique, l'acide palmitique-1.14 $\mathrm{C}$ et l'acide palmitique des graisses alimentaires. 


\section{RÉSULTATS}

\section{Essai no 1}

Les principaux résultats du premier essai font l'objet du tableau 3. La comparaison de l'activité spécifique du carbone de 1'acide palmitique vitellin à celle du précurseur se traduit par un rapport de 0,689 avec le régime lipidoprive, 0,187 et 0,453 pour le glucose et l'acide palmitique respectivement avec le régime gras. Dans ce tableau figure aussi un bilan du carbone radioactif dans les lipides de réserves et dans le vitellus exporté. Puisque l'on connaît la masse de précurseur ingérée, it

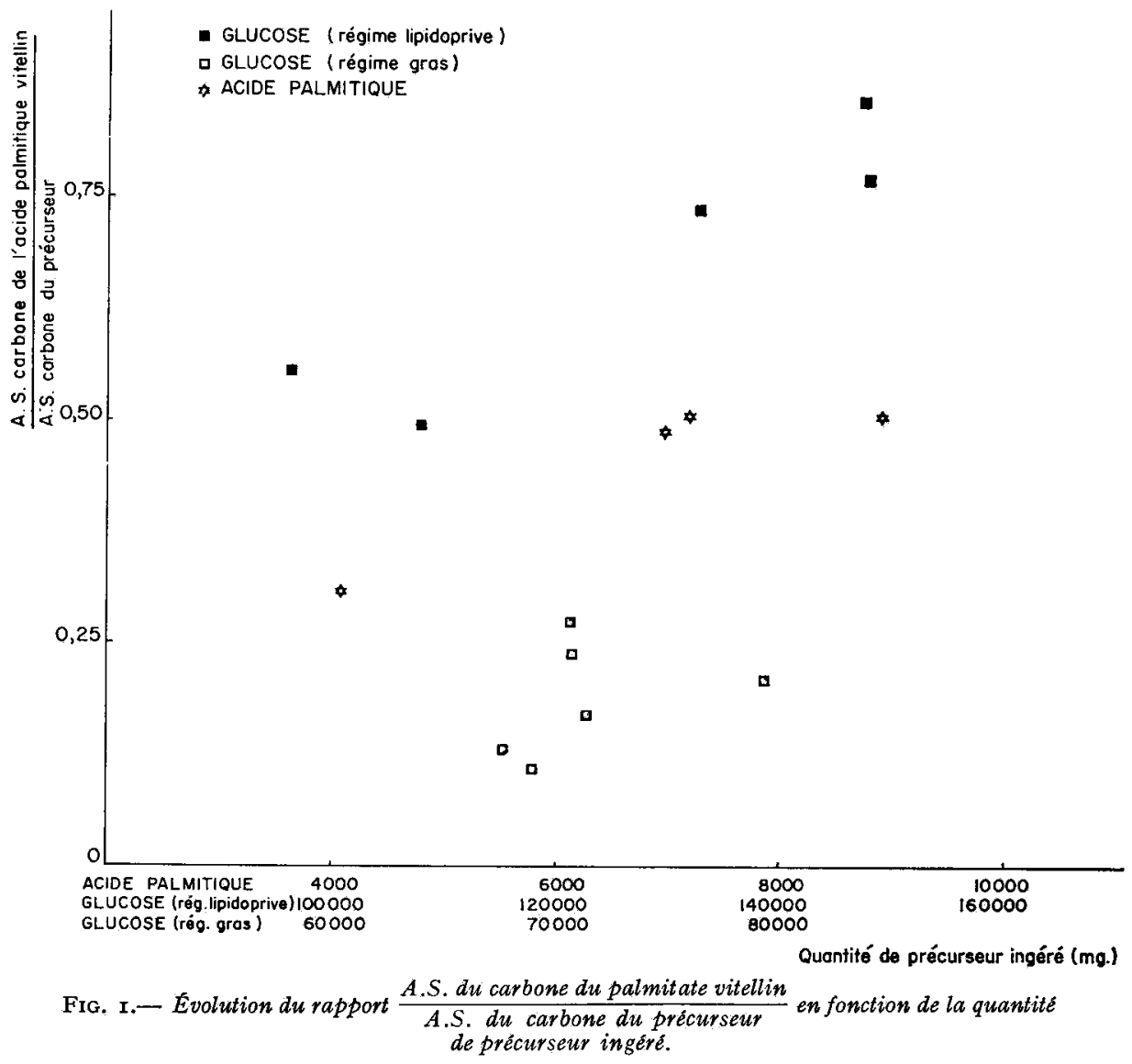

est possible d'estimer la quantité d'acides gras corporels et d'acides gras vitellins synthétisés en 48 heures à partir du précurseur considéré. On peut constater l'excellente utilisation de l'acide palmitique alimentaire dont plus de $25 \mathrm{p}$. roo sont incorporés soit aux acides gras des réserves adipeuses, soit aux acides gras de l'œuf. $\overline{\mathrm{I}} 1$ 
en est tout autrement du carbone du glucose dont une fraction beaucoup plus importante ne figure pas dans les acides gras synthétisés par 1'anima1; ce phénomène est spécialement prononcé dans le cas du régime gras. On remarque enfin que, en moyenne et à partir du précurseur (glucose ou acide palmitique), la Poule dépose sensiblement la même quantité de lipides dans ses réserves et dans son œuf lorsqu'elle ingère le régime gras. Au contraire, avec le régime lipidoprive, la Poule synthétise près de deux fois plus d'acides gras vitellins que d'acide gras de réserve.

Les résultats du tableau 3 sont des valeurs moyennes. L'étude des données individuelles permet de faire quelques observations supplémentaires. Ainsi, dans la figure $I$, on représente l'évolution du rapport

activité spécifique du carbone de l'acide palmitique vitellin activité spécifique du carbone du précurseur

en fonction de la quantité de précurseur consommée. Quel que soit le précurseur, on note une élévation du rapport à mesure que la quantité de précurseur consommée s'accroît. Toutefois, il semble qu'au-delà d'un certain niveau d'ingestion la valeur du rapport n'augmente plus.

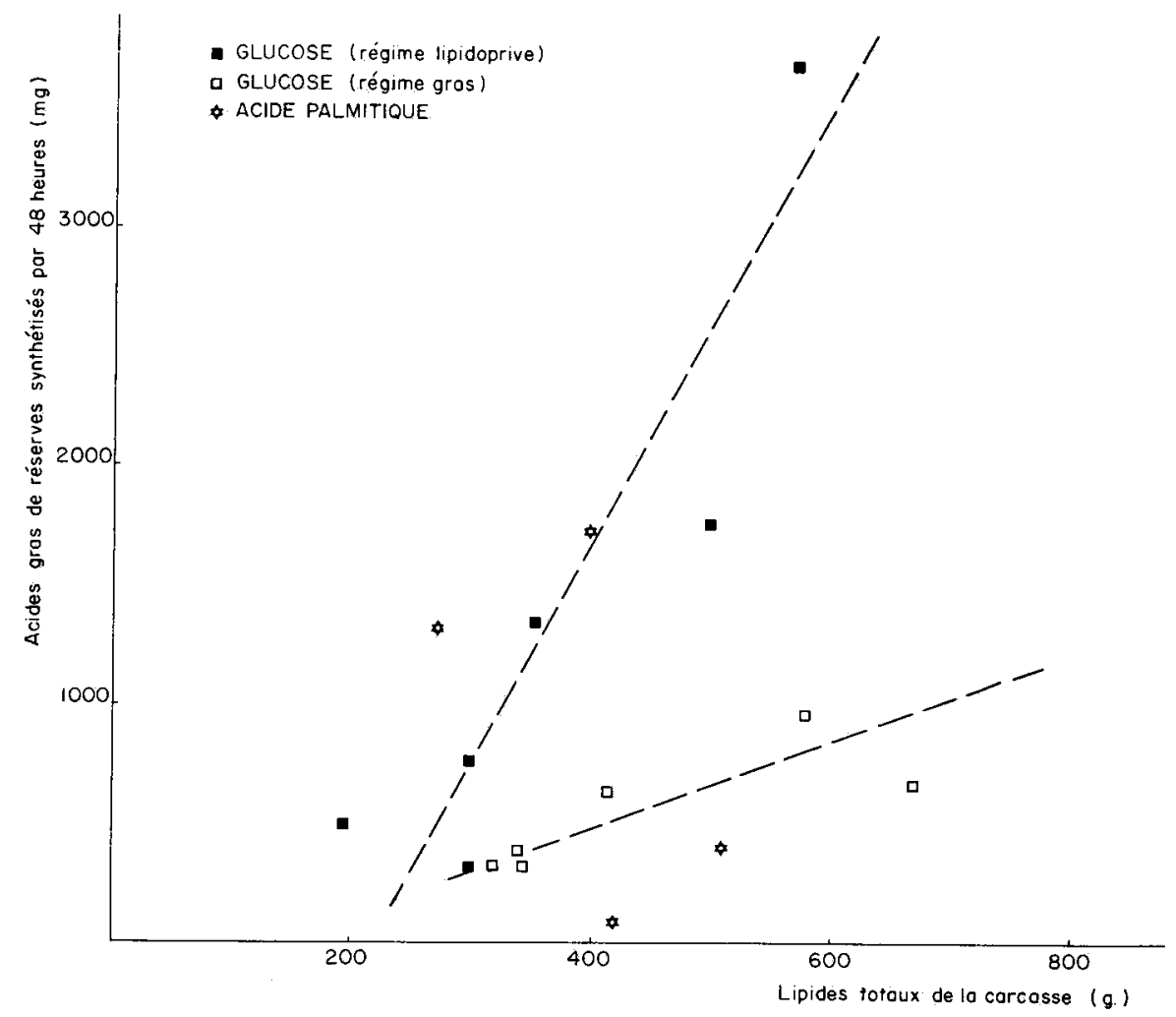

FrG. 2.- Relation entre la quantité d'acides gras déposés dans les réserves adipeuses en 48 heures et l'importance de ces réserves.

La figure 2 permet de relier l'intensité du dépôt journalier d'acides gras dans les tissus adipeux à l'importance de ces réserves. Dans le cas du glucose, on observe 
un dépôt journalier d'autant plus élevé que les réserves grasses de l'animal sont abondantes. Il en est tout autrement de l'acide palmitique. Son dépôt dans les graisses de réserves est indépendant de l'état d'engraissement.

Enfin, dans la figure 3 nous comparons la synthèse des acides gras des réserves à celle des acides gras vitellins. Quand le précurseur radioactif est le glucose, les intensités de ces synthèses évoluent dans le même sens. Une poule qui synthétise peu de lipides vitellins a également une lipogenèse réduite au niveau de ses réserves adipeuses et inversement. Cependant la relation est curvilinéaire ; le dépôt d'acides gras dans les tissus de réserves s'accroît beaucoup plus vite que la synthèse des lipides vitellins.

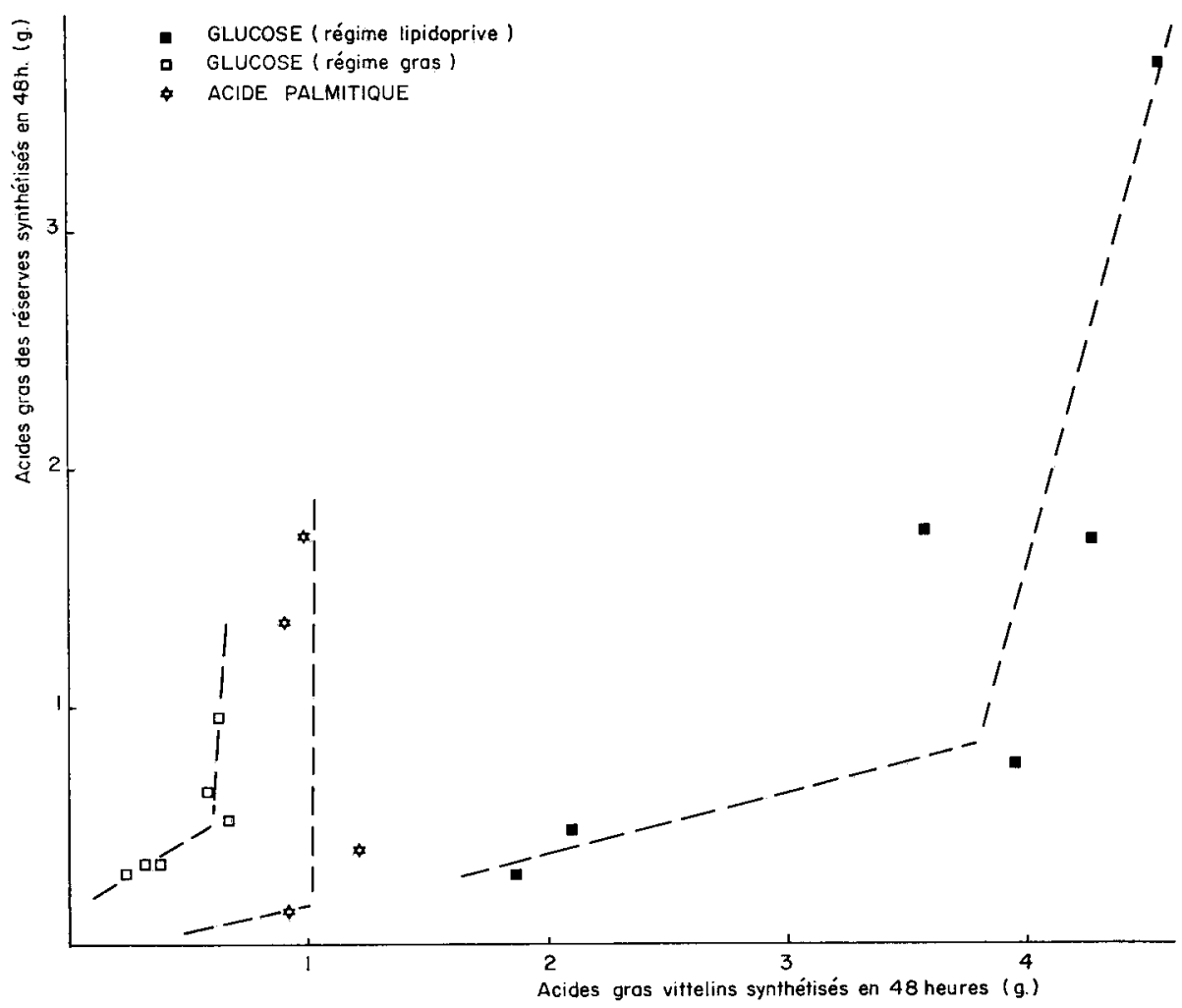

FIG. 3.- Corrélation entre la quantité d'acides gras déposés dans les qéserves apideuses et la quantité d'acides gras déposés dans l'ouf en 48 heures.

\section{Essai no 2}

Les résultats du second essai sont présentés dans le tableau 4 . Avec le glucose comme précurseur, la radioactivité se répartit dans les divers acides gras proportionnellement à l'importance pondérale de chacun d'eux, à l'exception de l'acide arachidonique. Ceci est particulièrement net pour le régime lipidoprive. Dans le cas du régime gras, les acides saturés sont relativement plus marqués que les acides désaturés. Avec chacun des acides gras précurseurs, on retrouve le maximum de 
B. LECLERCQ

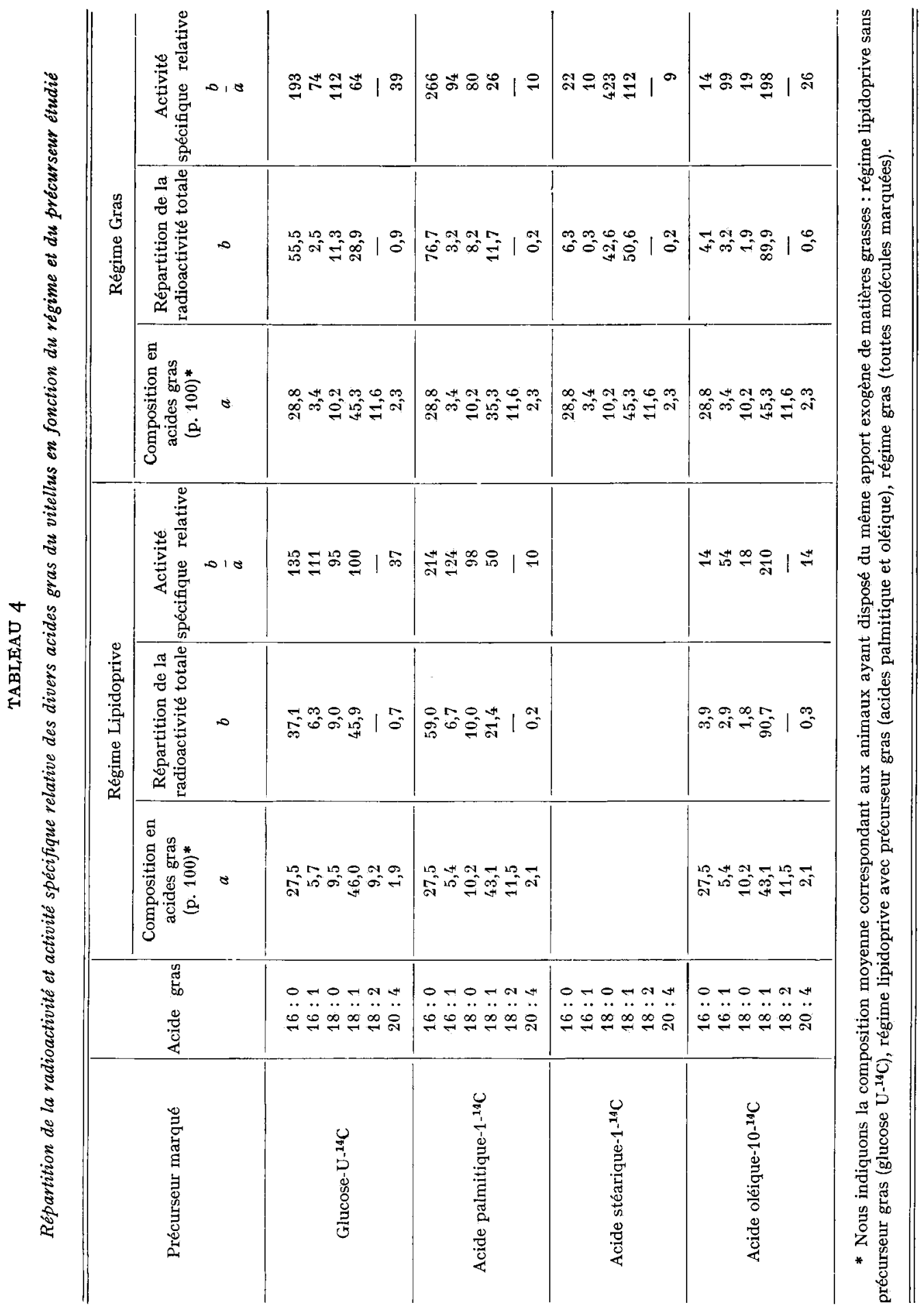


l'activité spécifique dans l'acide gras vitellin correspondant au précurseur ingéré. Cependant, une quantité appréciable de la radioactivité est retrouvée dans les acides gras dérivant du précurseur par désaturation ou élongation. Pour l'acide palmitique, il s'agit des acides palmitoléique, stéarique et oléique; pour l'acide stéarique, c'est l'acide oléique. Quand la poule ingère de l'acide oléique marqué, on retrouve $90 \mathrm{p}$. Ioo de la radioactivité dans cet acide gras au niveau du vitellus. On peut, par ailleurs, remarquer, qu'avec le régime enrichi en graisse, ces transformations sont moins prononcées, comme le révèlent les résultats relatifs à l'acide palmitique. Ennfin, l'activité spécifique de l'acide arachidonique est faible par rapport à celle des autres acides gras.

\section{DISCUSSION}

On peut, à partir de ces résultats, envisager d'étudier l'origine des acides gras de l'œuf en précisant l'importance d'une part de ce qui provient directement de l'aliment, d'autre part de ce qui provient des réserves corporelles. Cependant, il faut au préalable formuler quelques hypothèses qu'impose notre protocole expérimental. Tout d'abord, il est supposé que la destinée métabolique du glucose-U-14 $\mathrm{C}$ est la même que celle des autres glucides du régime, en particulier de l'amidon De ce point de vue, l'emploi d'antibiotique est utile puisqu'il limite l'importance d'une dégradation prématurée des sucres libres au niveau du jabot (SzyLIT et al., I965). Il faut ensuite admettre que 1'activité spécifique du précurseur est uniforme tout au long des 48 heures malgré un apport discontinu des molécules radioactives et le rythme propre de la consommation spontanée d'aliment de la poule. Enfin, on suppose qu'au cours d'une expérience aussi brève, la saturation des pools glucidiques et lipidiques du sang et du foie est atteinte en quelques heures et qu'à ces niveaux 1'équilibre isotopique est complètement installé au bout d'une douzaine d'heures (RIIS et HERSTAD, I967). Au contraire, le marquage du tissu adipeux étant infiniment plus lent, on peut penser que le retour d'acides gras marqués provenant des réserves est négligeable.

\section{I. - Origine alimentaire des acides gras vitellins}

La valeur du rapport entre l'activité spécifique du carbone de l'acide palmitique vitellin et l'activité spécifique du carbone du précurseur indique la part de l'acide palmitique du vitellus qui provient directement du précurseur alimentaire utilisé. Si cette valeur est nulle, ce précurseur ne participe pas à l'élaboration de l'acide palmitique. Si cette valeur atteint $I$, tout l'acide gras de l'œuf provient directement du précurseur étudié. Nos résultats suggèrent qu'environ $70 \mathrm{p}$. Ioo de 1'acide palmitique du régime provient des glucides et de l'acide palmitique alimentaires. Pour ce dernier, la valeur de 45,3 p. roo est inférieure à la réalité. En effet, elle correspond à la moyenne de quatre données parmi lesquelles trois sont situées entre 49 et $5 \mathrm{I}$ p. roo ; la dernière, égale à $3 \mathrm{I}, 2 \mathrm{p}$. IOO, correspond à une poule que l'ingestion de gélules a indisposée et qui, de ce fait, a réduit notablement sa consommation d'aliment au cours des 48 heures d'expérience. 
On remarque la contribution toute particulière des acides gras du régime à la vitellogenèse. Ainsi, pour les acides gras synthétisables par les tissus animaux, comme pour ceux qui ne le sont pas, la Poule utilise préférentiellement ceux du régime pour la synthèse de son œuf. A ce niveau, les remaniements entre acides gras sont limités, comme en témoignent les résultats du second essai. Ces interconversions correspondent surtout à un allongement de la chaîne aliphatique et à une désaturation, si bien, que l'acide oléique qui est très abondant et ne peut être ni allongé, ni désaturé, ne contribue que très peu à la synthèse d'autres acides gras Ainsi, on s'explique que toute surcharge alimentaire en acides gras synthétisables par l'animal n'ait aucun effet sur la composition en acides gras de l'œuf (CALET, I959) excepté pour l'acide oléique (DONALDSON, I966; LECLERCQ, I967). La répartition de la radioactivité entre les différents acides gras lors de l'emploi du glucose radioactif peut s'interpréter de la même façon. Avec le régime gras, l'activité spécifique des acides saturés est supérieure à celle des acides insaturés; ce qui peut s'expliquer par une utilisation préférentielle des acides désaturés de la matière grasse du régime.

Si près de $70 \mathrm{p}$. Ioo de l'acide palmitique de l'œuf provient directement des glucides ou de l'acide palmitique de l'aliment, le reste (30 p. Ioo) tire son origine soit d'autres composants alimentaires, soit des réserves adipeuses. La participation d'autres composants du régime, comme les protéines, doit être infime, étant donné leur faible teneur dans le régime et leur destinée préférentielle pour la protéinogenèse. On peut donc penser que la majeure partie des 30 p. Ioo non expliqués représentent la contribution du tissu adipeux.

\section{2. - Synthèse et utilisation des réserves adipeuses pour la vitellogenèse}

Les données de la figure I suggèrent qu'en cas d'ingestion insuffisante d'aliment, la Poule dilue le carbone alimentaire d'origine glucidique ou lipidique par une autre source de carbone non radioactif qui doit être essentiellement $\mathbf{d u}$ carbone d'origine adipeuse. La poule pourrait donc pallier une insuffisance modérée et temporaire de composants énergétiques alimentaires. Dans notre essai, trois poules, ayant mal supporté les manipulations fréquentes imposées par le protocole, ont sousconsommé et maintenu cependant leur synthèse d'acides gras vitellins en prélevant sur leurs tissus.

Cependant, dans des conditions nutritionnelles normales, un accroissement de la lipolyse ne doit guère intervenir pour le maintien de la ponte. C'est ce que suggère la figure 2 ; les réserves grasses totales de la carcasse sont en effet proportionnelles à la quantité d'acides gras déposés à ce niveau, en 48 heures. On peut affirmer que les poules les plus grasses sont celles qui déposent le plus d'acides gras dans leur tissu adipeux parce que l'intensité de la lipolyse est moins élevée que celle de la lipogenèse.

Enfin, il faut bien constater un parallélisme entre intensité du dépôt d'acides gras dans l'œuf et intensité du dépôt dans les réserves (fig. 3). Il y aurait donc, corrélation entre ces deux processus lipogénétiques. Dans une certaine mesure, les animaux les plus enclins à une synthèse élevée d'acides gras vitellins sont également ceux chez lesquels l'adiposité tend à se développer avec la plus forte intensité (JAAP et Clancy, I968). Toutefois, au cours de l'ovogenèse, des processus autres que la lipogenèse peuvent devenir limitants (protéinogenèse, synthèse de la coquille...) de sorte 
qu'au-delà d'un certain niveau de lipogenèse, la Poule détourne au profit de ses réserves des proportions de plus en plus élevées d'acides gras.

Dans notre essai, ce phénomène semble plus prononcé avec le régime gras qu'avec le régime lipidoprive. En effet, en supposant que l'utilisation des acides gras alimentaires soit semblable à celle de l'acide palmitique, on déduit que les matières grasses du régime ont permis la synthèse de 4 ,I5 $\mathrm{g}$ d'acides gras vitellins $\left(0,97 \times \frac{\text { IO0 }}{24, \mathrm{I}}\right)$ et de $3,70 \mathrm{~g}$ d'acides gras des réserves adipeuses $\left(0,89 \times \frac{\mathrm{IO0}}{24, \mathrm{I}}\right)$. Au total, dans le cas du régime gras, les poules déposent à partir des glucides et des graisses de leur aliment $4,63 \mathrm{~g}(4, \mathrm{I} 5+0,48)$ et $4,25 \mathrm{~g}(3,70+0,55)$ d'acides gras respectivement dans leur vitellus et leur tissu de réserves, alors qu'avec le régime lipidoprive, ces dépôts ne sont que de 3,40 et I,46 g. La différence entre les deux régimes est faible pour la synthèse d'acide gras vitellin et peut s'expliquer par des intensités de ponte un peu différentes. Il en est tout autrement au niveau du tissu adipeux puisque dans le cas du régime gras, l'intensité est trois fois supérieure à celle du régime lipidoprive. L'ingestion de constituants énergétiques étant semblable avec l'un et l'autre régimes, on doit conclure que leur utilisation pour la lipogenèse est supérieure lorsqu'ils proviennent des graisses. La vitellogenèse ne pouvant s'accroître, c'est vers le tissu adipeux que sont dirigés les acides gras en excès.

En définitive, ces résultats s'expliquent par le rôle essentiel que joue le foie dans la synthèse des lipides chez les Oiseaux (Goodridge et BAII, r967). Recevant directement les produits de la digestion en l'absence de système lymphatique développé (NoyAN et al., I964), responsable de la majeure partie des synthèses lipidiques de l'organisme, le foie élabore des lipides vitellins dont les éléments proviennent directement des constituants du régime, avec une préférence très marquée pour les acides gras. Le tissu adipeux est essentiellement un lieu de réserve dans lequel, en conditions normales, le dépôt revêt une importance supérieure à l'utilisation. L'intensité de ce dépôt est en partie liée à celle de la vitellogenèse. En ce sens, un certain état d'engraissement est inéluctablement lié à la ponte. Cependant, en cas de lipogenèse trop intense, seule la quantité d'acides gras déposés dans les réserves adipeuses s'accroît. Ce dépôt inutile compromet dans l'immédiat une utilisation efficace de l'aliment et à plus ou moins long terme, l'aptitude de la pondeuse. L'alimentation rationnelle de la Poule en ponte doit permettre de limiter ces dépôts excessifs. On conçoit cependant la difficulté d'une telle opération qui ne doit pas réduire l'intensité des synthèses vitellines.

Rę̧u pour publication en janvier 1972.

SUMMARY

STUDY OF THE BIOSYNTHESIS AND UTILIZATION OF FATTY ACIDS IN THE LAYER

In a first experiment, the proportion of palmitic acid in the egg coming directly from carbohydrates or from palmitic acid in the feed, was determined according to whether the hens ate a lipid-deficient diet or a fat diet ( 5 p. fat content). The animals were given oral doses of either glucose U-C14 or palmitic acid $\mathrm{I}_{-} \mathrm{C}^{14}$ for $4^{8}$ hours. The yolk synthesized during the last 24 hours 
was determined by lipophilic coloring eaten by the hen. A comparison of the specific carbon activity of yolk palmitic acid with that of the precursor carbon (ratio $\frac{e}{d}$ in table 3 ) shows that about $70 \mathrm{p}$. roo of egg palmitic acid comes directly from carbohydrates and lipids in feeds. The hen preferentially uses fatty acids in the diet for yolk lipid synthesis. Interconversions between fatty acids are limited; they occur mainly in the way of desaturation and of aliphatic chain elongation. These interconversions are diminished when a fat diet is eaten (table 4).

When there is deficient carbohydrate or lipid intake, the value of the ratio $\frac{e}{d}$ decreases (figure I), thus suggesting a dilution of the feed carbon by a non-radioactive source, probably from adipose tissue. The hen thus may overcome a moderate temporary deficiency of energetic components in the feed. The mass of storage fat in the carcass is directly proportional to the quantity found in the tissues (fig. 2). Thus, it seems that in normal nutritional conditions, fatty acid deposit in adipose tissue is much greater than its mobilization.

There is a curvilinear relation between the intensity of yold fatty acid synthesis and that of the deposit in adipose tissue (fig. 3). Animals with a tendency to intense vitellogenesis are also those which tend to develop great adiposity. When lipogenesis is too intense, however, the hen stores away larger and larger amounts of fatty acid.

\section{RÉFÉRENCES BIBLIOGRAPHIQUES}

Almouist H. J., Lorenz F. W. h, Burmeister B. R., r934. Relation of depot fat to egg yolk fat laying hens. J. Biol. Chem., 106, 365-371,

Balnave D., Brown W. O., I 968 . A study of the separate effects of energy intake and dietary corn oil on egg production and egg size in EFA deficient thens fed a semi-purified diet. Poult. Sci., 47, 1212I219.

CALET C., I959. Données nouvelles sur l'influence de l'alimentation sur la quantité et la composition des lipides de l'œuf. Ann. Nutr. Alim., 13, A 163-A 205.

Donalson W. E., I966. Fatty acid interconversions in laying hens. Poult. Sci., 45, 473.

Feigenbaum A. S., Fisher H., I959. The influence of dietary fat on the incorporation of fatty acids into body and egg fat of the hen. Arch. Bioch. Biophys., 79, 302-306.

Goodridge A. G., Ball E. G., I967. Lipogenesis in the pigeon : in vivo studies. Am. J. Physiol., 213, $245-249$.

Heald P. J., Badman H. G., I963. Lipid metabolism and the laying hen. I. Plasma free fatty acids and the onset of laying. Bioch. Biophys. Acta, 70, 381-388.

Ivorec-Szylit O., Mercier C., Raibaud P., Calet C., 1965. Contribution à l'étude de la dégradation des glucides dans le jabot du coq. III. Influence du taux de glucose du régime. C. R. Acad. Sci., 261, 320I-3203.

JAAP R. G., CLANCY J. A., 1968. Reproductive idiosyncrasies of the broiler pullet. Proc, 3rd European Poultry Conference. Jerusalem.

Kaufman H. P., Mankel A., I967. Ủber trans-Lipoide : Die Lipoide des Huhnerei. Dutters. Fette Seif. Anstrich., 69, 107-1 I0.

Leclerco B., BLUM J.-C., JacQuot R., 1965. Étude de l'utilisation de l'acide élaïdique en cours de l'ovogenèse de Gallus gallus : son incorporation dans les graisses de réserves et dans les lipides de l'œuf pondu. C. R. Acad. Sci., 261, 3197-3200.

Leclerco B., r967. Utilisation de l'acide élaïdique pour l'étude du métabolisme des lipides chez la Poule pondeuse. Arch. Sci., physiol., 21, 299-322.

Murty N. L., Rerser R., 196r. Influence of graded levels of dietary linoleic and linolenic acids on the fatty acid composition of hens'eggs. J. Nutr., 75, 287-294.

Noyan A., Lossow W. J., Brot N., Chaikoff I. L., I964. Pathways and form of absorption of palmitic acid in the chicken. J. Lipid Res., 5, 538-54I.

O'HeA E. K., Leveille G. A., I968. Lipogenesis in isolated adipose tissue of the domestic chick (Gallus domesticus). Comp. Biochem. Physiol., 26, I I I-I20.

REISER R., 1950. Fatty acid changes in the yolk of hens on a fat free and a cottonseed oil ration. $J$. Nutr., 40, 429-435.

Rirs P. M., Herstad O., 1967. Kinetics of glucose metobolism in the normal laying hen. Acta Agric. Scand., 17, 3-12.

Wheeler P., Peterson D. W., Michaels G. D., I959. Fatty acid distribution in egg yolk as influenced by type and level of dietary fat. J. Nutr., 69, 253-260. 